\title{
Single-electron transfer between two donors in nanoscale thin silicon-on-insulator field-effect transistors
}

\author{
Earfan Hamid, Daniel Moraru, Juli Cha Tarido, Sakito Miki, Takeshi Mizuno, and \\ Michiharu Tabe ${ }^{a}$ \\ Research Institute of Electronics, Shizuoka University, 3-5-1 Johoku, Nakaku, Hamamatsu 432-8011, Japan
}

(Received 13 September 2010; accepted 2 December 2010; published online 28 December 2010)

\begin{abstract}
We describe single-electron transfer between two donors in thin silicon-on-insulator field-effect transistors with phosphorus-doped channel. At low temperatures, single-electron tunneling through one donor can be identified in source-drain current/gate voltage measurements as a single current peak. On this peak, we observed hysteresis most likely as a signature of single-electron transfer with another donor. The origin of single-electron transfer is related to different intensities of coupling between each donor and the interface, as evidenced from simulations. It was found that donor-interface coupling is essential for the energetic transfer of a single-electron location within the two-donor system. (C) 2010 American Institute of Physics. [doi:10.1063/1.3530442]
\end{abstract}

Individual dopant atoms play a key role in the electrical characteristics of nanoscale field-effect transistors (FETs). ${ }^{1}$ In fact, it was revealed that, at low temperatures, a single isolated dopant works as a quantum dot (QD), mediating single-charge tunneling from source to drain. ${ }^{2,3}$ Even when a large number of donors are found in nanoscale-channel silicon-on-insulator (SOI) FETs, single-electron transport is still mediated by only one or a few donors. ${ }^{4}$ In these systems, it is essential to clarify donor-donor interaction, which can be useful for applications such as charge-based quantum computing ${ }^{5}$ and single-electron turnstiles. 6,7

In this work, we demonstrate single-electron transfer between two donors in thin SOI-FETs. This is evidenced by the hysteresis on the first observable current peak in the currentvoltage characteristics. The findings are supported by simulation results for two parallel donor-QDs with limited occupancy. We show that single-electron transfer between two donors is only possible if the gate capacitances of the two donors cross over. By considering the behavior of donors near interfaces, ${ }^{8-11}$ we introduce a concept of voltagedependent donor-gate capacitance to explain single-electron transfer.

The devices investigated in this work are shown in Fig. 1(a). The SOI layer is doped with phosphorus. Doping concentration, estimated from secondary ion mass spectroscopy, is $N_{d} \approx 1 \sim 3 \times 10^{18} \mathrm{~cm}^{-3}$, equivalent to an average interdonor distance of $7-10 \mathrm{~nm}$. The channel is $10-\mathrm{nm}$-thick and less than 50-nm-wide. The channel area is connected to gradually wider $\mathrm{Si}$ pads that work as effective source and drain. A wide $\mathrm{Al}$ front gate is deposited over a thermally grown 10-nm-thick $\mathrm{SiO}_{2}$ layer.

Figure 1(b) shows an electronic potential landscape, simulated for a random arrangement of donors. In these simulations, the channel is assumed to be depleted, so the potential is given by the superposition of the Coulomb potentials of all ionized donors. ${ }^{4,12,13}$ This situation corresponds to the onset of conduction, when the gate voltage aligns the lowest channel potential with the source Fermi level. The fine potential valleys are ascribed to individual donors. In

\footnotetext{
a) Author to whom correspondence should be addressed. Electronic mail: romtabe@ rie.shizuoka.ac.jp.
}

some cases, we encounter the following situation: one donor $\left(D_{1}\right)$ is the steppingstone for conduction in the $x$ direction, while a second donor $\left(D_{2}\right)$ may work as a single-electron trap.

We measured the source-drain current $\left(I_{\mathrm{SD}}\right)$-front gate voltage $\left(V_{\mathrm{G}}\right)$ characteristics at low temperatures $(\sim 15 \mathrm{~K})$. All devices invariably exhibit irregular current oscillations, such as shown in Fig. 2(a), indicating single-electron tunneling transport through donor-induced QDs. ${ }^{4,6,7}$

We focused on the first observable peak, which corresponds to the conduction onset. It is known that a symmetric and isolated peak indicates conduction through a single donor. ${ }^{4}$ This first peak is isolated from the following peaks by a wide $V_{\mathrm{G}}$ range. Two examples of $I_{\mathrm{SD}}-V_{\mathrm{G}}$ characteristics, in Figs. 2(b) and 2(c), show first current peaks due to single-electron tunneling via one donor.

In order to observe charging in the vicinity of this donor, the characteristics were measured for a limited $V_{\mathrm{G}}$ range around the first current peak. $V_{\mathrm{G}}$ was swept upward and, successively, downward with a sweep rate of $1 \mathrm{mV} / \mathrm{s}$. In Fig. 2(b), abrupt current jumps can be observed on the first peak: a jump up in the upward sweep, at $V_{\mathrm{G}}=1.345 \mathrm{~V}$, followed by a jump down in the downward sweep, at $V_{\mathrm{G}}=1.335 \mathrm{~V}$. These features produce a fine but reproducible hysteresis, which can be ascribed to trapping and detrapping of a singleelectron in a memory dot. The nature of the memory dot can be clarified from time-dependent $I_{\mathrm{SD}}$ measurements, with $V_{\mathrm{G}}$ set inside the hysteresis region $\left(V_{\mathrm{G}}=1.34 \mathrm{~V}\right)$. A random telegraph signal (RTS) can be resolved, as shown in the inset. The signal contains mainly two current levels, suggesting a two-level trap. It is natural to assume that the trap is a donor,

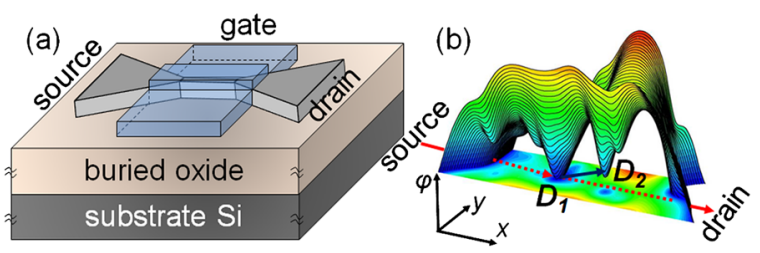

FIG. 1. (Color online) (a) Schematic view of doped-nanowire SOI-FETs. (b) Potential profile simulated for a random distribution of ionized donors; two neighboring donors are indicated. 


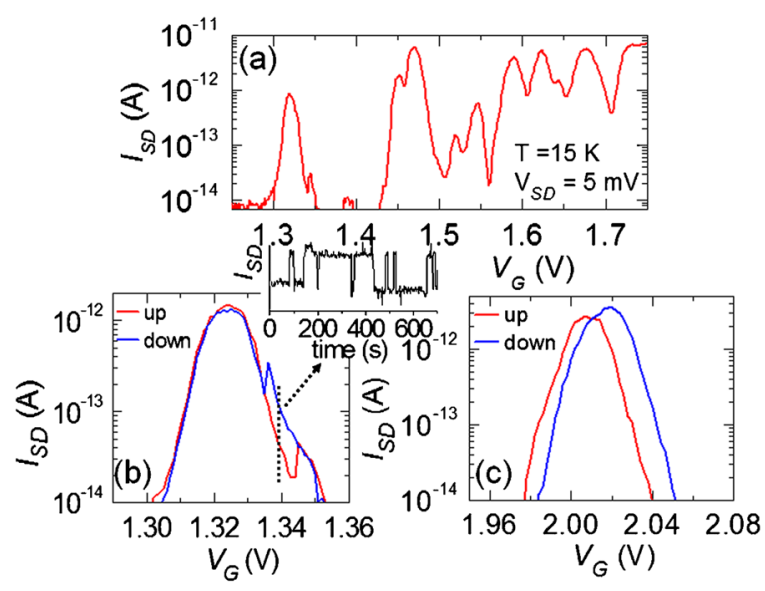

FIG. 2. (Color online) (a) $I_{\mathrm{SD}}-V_{\mathrm{G}}$ characteristics for wide $V_{\mathrm{G}}$ range, showing a sequence of irregular current oscillations. [(b) and (c)] Measurements of the first peak by successively sweeping $V_{\mathrm{G}}$ upward and downward for two different devices. Inset: $I_{\mathrm{SD}}$-time trace at $V_{\mathrm{G}}=1.34 \mathrm{~V}$, corresponding to the dashed line in (b). For all measurements, $T=15 \mathrm{~K}$ and $V_{\mathrm{SD}}=5 \mathrm{mV}$.

which can only have two states $\left(D^{+}\right.$and $\left.D^{0}\right)$. The $D^{-}$state, corresponding to a second electron being added to a donor, is only several meV below the conduction band edge ${ }^{14}$ and is not expected to be observed in our measurements $(T>15 \mathrm{~K})$.

For the device shown in Fig. 2(c), current jumps cannot be observed on the current peak, but charging is evident from the displacement between upward and downward curves.

Both $I_{\mathrm{SD}}-V_{\mathrm{G}}$ characteristics presented here illustrate thus charging and discharging in a two-donor system. One donor works as a stepping stone for conduction, giving rise to the first current peak. Another donor modulates the current by trapping and detrapping one electron. This second donor does not contribute as a conduction path, since we observe a simple shift of the original peak. Charging occurs on the current peak [Fig. 2(b)] or off the current peak [Fig. 2(c)], as will be also shown in the simulation results in Fig. 4. This suggests that conduction through the first donor and trapping in the second donor are correlated. This is a strong indication that trapping and detrapping occur by single-electron transfer between the two donors, although other trapping paths, such as direct injection from one terminal, cannot be completely excluded.

In simulation, we model the two-donor system as two parallel QDs, as shown in Fig. 3(a). Single-electron transport simulations are performed within the orthodox Coulomb blockade theory. The two donors are physically separated by a tunnel barrier and, therefore, a tunnel junction is inserted between them. Donor $D_{1}$, located close to the center of the channel in horizontal and vertical directions, ${ }^{4}$ works as conduction path and is coupled to source and drain by tunnel junctions $\left(C_{\mathrm{S} 1}\right.$ and $\left.C_{\mathrm{D} 1}\right)$. Donor $D_{2}$ is displaced from the center toward the edge of the channel, where the potential barriers in the source-drain direction are higher for small voltages. ${ }^{4}$ Therefore, this donor is coupled to source and drain via nontunnel capacitors $\left(C_{\mathrm{S} 2}\right.$ and $\left.C_{\mathrm{D} 2}\right)$. Other donors are also present in the device channel, but their energies are higher than the Fermi energy. In consequence, their effect can be basically incorporated in these tunnel barriers. The two donor-QDs are commonly coupled to the same gate.

Our purpose is to reproduce the hysteresis in the $I_{\mathrm{SD}}$ $-V_{\mathrm{G}}$ characteristics, by using the circuit shown in Fig. 3(a).
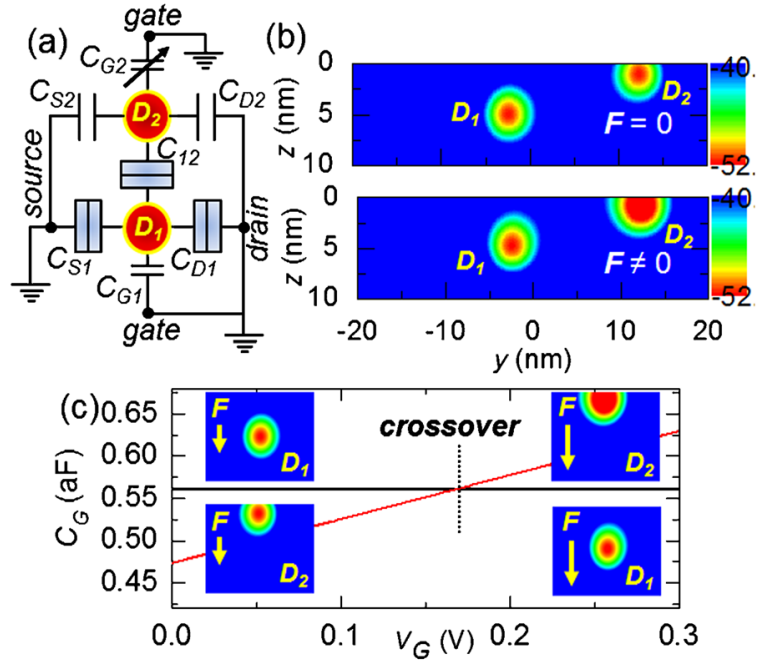

FIG. 3. (Color online) (a) Equivalent circuit of two parallel coupled donorQDs with variable gate capacitance. (b) Cross-sectional potential landscape for two donors without (top) and with (bottom) applied electric field $\boldsymbol{F}$. (c) $C_{\mathrm{G}}-V_{\mathrm{G}}$ dependence reflecting the donor-interface coupling under increasing electric field for the two donors: deeper donor (horizontal line) and superficial donor (inclined line).

However, this hysteresis should only involve the two states of each donor, i.e., one electron is transferred from a neutral donor to an ionized donor. When the donors are considered as QDs with fixed gate capacitances, single-electron transfer cannot be achieved unless other assistant electrons are involved in the process. In previous reports of two-classicaldot circuits with constant gate capacitances, ${ }^{15,16}$ hysteresis observed as a signature of single-electron memory operation involves more than one elementary charge, although not explicitly described. For a two-donor system, only one electron can be involved in the transfer process. This can only be explained if the gate capacitances of the two donors cross over at a certain gate voltage. This crossover induces an energetic transfer of the electron location in the two-donor system and it can only be realized by considering voltagedependent donor-gate capacitances, as we incorporate in our simulations.

In addition, voltage-dependent donor-gate capacitance is reasonable for donors in thin Si channels. Figure 3(b) shows the $y-z$ cross-sectional potential landscape for an arrangement of two donors in a thin $\mathrm{Si}$ channel. Two cases are shown: without and with an electric field $\boldsymbol{F}$ applied vertically (along $z$ axis). Current flows in the $x$ direction through donor $D_{1}$. This donor, located relatively far from the interface (5 $\mathrm{nm}$ here), is not significantly affected by the electric field. On the other hand, the second donor $D_{2}$ is close to the interface (1 $\mathrm{nm}$ here). For such superficial donor, it is known that the potential expands at the interface under electric field. ${ }^{8-11}$ The cross-sectional area toward the gate and, implicitly, the donor-gate capacitance $\left(C_{\mathrm{G}}\right)$ increase with electric field (i.e., voltage $V_{\mathrm{G}}$ ). Therefore, for such a donor, $C_{\mathrm{G}}$ is continuously changing with $V_{\mathrm{G}}$.

Figure 3(c) shows the gate capacitances for the two donors with fixed depths as a function of $V_{\mathrm{G}}$, as considered in the following simulations. The gate capacitance $C_{\mathrm{G} 1}$ of the deeper donor $D_{1}$ is practically constant, while the gate capacitance $C_{\mathrm{G} 2}$ of the superficial donor $D_{2}$, changes with $V_{\mathrm{G}}$. The slope of this change reflects the donor depth below interface. At small $V_{\mathrm{G}}, C_{\mathrm{G} 2}$ can be even smaller than $C_{\mathrm{G} 1}$ due 


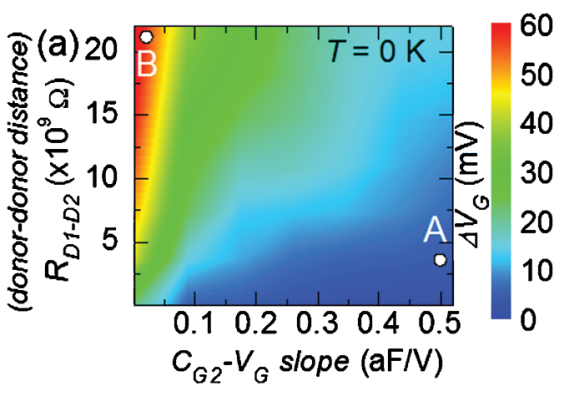

(donor-interface distance)

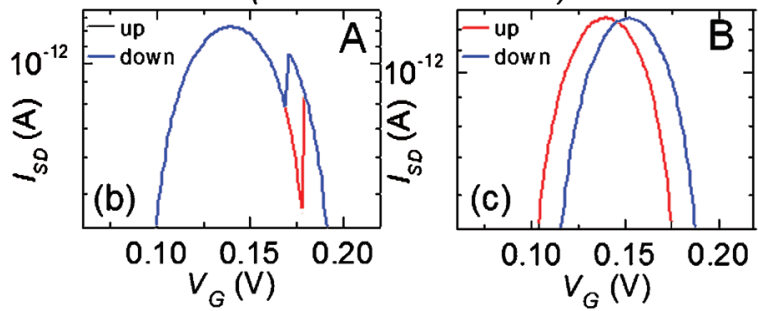

FIG. 4. (Color) (a) Contour plot of the voltage shift $\Delta V_{\mathrm{G}}$ (difference between the voltages at which trapping and detrapping occur) as a function of $C_{\mathrm{G} 2}-V_{\mathrm{G}}$ slope and donor-donor tunnel resistance. [(b) and (c)] Simulated $I_{\mathrm{SD}}-V_{\mathrm{G}}$ characteristics (upward and downward $V_{\mathrm{G}}$ ramping) for two cases, indicated in (a): point A (b) and point B (c).

to the reduced area toward the gate. A crossover between the gate capacitances of the two donors occurs at a certain $V_{\mathrm{G}}$. We suggest that this capacitive crossover is crucial in determining the energetic transfer of an electron between the two donors. For small $V_{\mathrm{G}}$ 's, when $C_{\mathrm{G} 2}<C_{\mathrm{G} 1}$, it is energetically favorable for the electron to occupy donor $D_{1}$. After the crossover point, when $C_{\mathrm{G} 2}>C_{\mathrm{G} 1}$, the system energetically favors the electron transfer to donor $D_{2}$. This can explain the origin of the single-electron transfer.

We observed, however, that single-electron transfer occurs with a delay relative to the crossover voltage, which gives rise to a hysteresis between single-electron trapping and detrapping events. We monitored the dependence of the width of this hysteresis, $\Delta V_{\mathrm{G}}$, as a function of two factors, as shown in Fig. 4(a): the trap donor's $C_{\mathrm{G}}-V_{\mathrm{G}}$ slope and the interdonor resistance $R_{\mathrm{D} 1-\mathrm{D} 2}$. The slope can be correlated with donor-interface distance, as explained above (see Fig. $3)$. Interdonor resistance can be correlated with the donordonor distance, i.e., interdonor barrier width. Both these parameters significantly affect the hysteresis width.

Two opposite donor arrangements are indicated by points $\mathrm{A}$ and $\mathrm{B}$. The simulated $I_{\mathrm{SD}}-V_{\mathrm{G}}$ characteristics for these configurations are shown in Figs. 4(b) and 4(c). For Fig. 4(b), donors are close to each other (small $R_{\mathrm{D} 1-\mathrm{D} 2}$ ) and the trap donor is close to interface (large $C_{\mathrm{G} 2}-V_{\mathrm{G}}$ slope). In this case, electron transfer occurs near the crossover $V_{\mathrm{G}}$, pro- ducing a fine hysteresis, similar to the experimental observation in Fig. 2(b). For Fig. 4(c), which corresponds to donors far from each other (large $R_{\mathrm{D} 1-\mathrm{D} 2}$ ) and the trap donor relatively far from interface (small $C_{\mathrm{G} 2}-V_{\mathrm{G}}$ slope), electron transfer is significantly delayed relative to the crossover $V_{\mathrm{G}}$. Thus, the current peak is totally shifted, similarly to the results shown in Fig. 2(c). The experimental results can, thus, be ascribed to these two different donor arrangements. From preliminary simulations, we also found that interdonor capacitance can assist in further separating the current peaks. In addition, by considering injection via a donor-induced multiple-tunnel-junction, an energy-based memory effect may be achievable. This work is under way.

In summary, we demonstrated single-electron transfer between two donors, evidenced by the hysteresis on the first current peak in thin SOI-FETs. We showed that the hysteresis is affected by the donor depth and donor-donor distance. These results can be useful in designing novel applications utilizing donor-donor interaction.

This work was partially supported by KAKENHI Grants-in-Aid for Scientific Research (Grant Nos. 18063010 and 20246060).

${ }^{1}$ A. Asenov, Nanotechnology 10, 153 (1999).

${ }^{2}$ H. Sellier, G. P. Lansbergen, J. Caro, S. Rogge, N. Collaert, I. Ferain, M. Jurczak, and S. Biesemans, Phys. Rev. Lett. 97, 206805 (2006).

${ }^{3}$ Y. Ono, K. Nishiguchi, A. Fujiwara, H. Yamaguchi, H. Inokawa, and Y. Takahashi, Appl. Phys. Lett. 90, 102106 (2007).

${ }^{4}$ M. Tabe, D. Moraru, M. Ligowski, M. Anwar, R. Jablonski, Y. Ono, and T. Mizuno, Phys. Rev. Lett. 105, 016803 (2010).

${ }^{5}$ L. C. L. Hollenberg, A. S. Dzurak, C. Wellard, A. R. Hamilton, D. J. Reilly, G. J. Milburn, and R. G. Clark, Phys. Rev. B 69, 113301 (2004). ${ }^{6}$ D. Moraru, Y. Ono, H. Inokawa, and M. Tabe, Phys. Rev. B 76, 075332 (2007).

${ }^{7}$ D. Moraru, M. Ligowski, K. Yokoi, T. Mizuno, and M. Tabe, Appl. Phys. Express 2, 071201 (2009).

${ }^{8}$ A. S. Martins, R. B. Capaz, and B. Koiller, Phys. Rev. B 69, 085320 (2004).

${ }^{9}$ M. J. Calderón, B. Koiller, and S. Das Sarma, Phys. Rev. B 75, 125311 (2007).

${ }^{10}$ G. P. Lansbergen, R. Rahman, C. J. Wellard, I. Woo, J. Caro, N. Collaert, S. Biesemans, G. Klimeck, L. C. L. Hollenberg, and S. Rogge, Nat. Phys. 4, 656 (2008).

${ }^{11}$ R. Rahman, G. P. Lansbergen, S. H. Park, J. Verduijn, G. Klimeck, S Rogge, and L. C. L. Hollenberg, Phys. Rev. B 80, 165314 (2009).

${ }^{12}$ M. Ligowski, D. Moraru, M. Anwar, T. Mizuno, R. Jablonski, and M. Tabe, Appl. Phys. Lett. 93, 142101 (2008).

${ }^{13}$ G. J. Evans, H. Mizuta, and H. Ahmed, Jpn. J. Appl. Phys., Part 1 40, 5837 (2001)

${ }^{14}$ M. Taniguchi and S. Narita, Solid State Commun. 20, 131 (1976).

${ }^{15}$ K. Yano, T. Ishii, T. Hashimoto, T. Kobayashi, F. Murai, and K. Seki, IEEE Trans. Electron Devices 41, 1628 (1994).

${ }^{16}$ A. Fujiwara, Y. Takahashi, K. Murase, and M. Tabe, Appl. Phys. Lett. 67, 2957 (1995) 